Research Paper

\title{
Exosomes Secreted by Human-Induced Pluripotent Stem Cell-Derived Mesenchymal Stem Cells Repair Critical-Sized Bone Defects through Enhanced Angiogenesis and Osteogenesis in Osteoporotic Rats
}

\author{
Xin $\mathrm{Qi}^{1^{*}}$, Jieyuan Zhang ${ }^{1,2^{*}}$, Hong Yuan ${ }^{3}$, Zhengliang $\mathrm{Xu}^{1}$, Qing $\mathrm{Li}^{2}$, Xin $\mathrm{Niu}^{2}$, Bin $\mathrm{Hu}^{2}$, Yang Wang ${ }^{2}$, \\ Xiaolin $\mathrm{Li}^{1}{ }^{\square}$ \\ 1. Department of Orthopaedic Surgery, Shanghai Jiao Tong University Affiliated Sixth People's Hospital, Shanghai, China. \\ 2. Institute of Microsurgery on Extremities, Shanghai Jiao Tong University Affiliated Sixth People's Hospital, Shanghai, China \\ 3. Department of Dermatology, Zhongshan Hospital, Fudan University, Shanghai, China. \\ *Xin Qi and Jieyuan Zhang are co-first authors of this article. \\ $\square$ Corresponding authors: Yang Wang (e-mail: wangy63cn@126.com), Xiaolin Li (e-mail: lixiaolin@sjtu.edu.cn) Address: Department of Orthopaedic Surgery, \\ Shanghai Jiao Tong University Affiliated Sixth People's Hospital, 600 Yishan Road, Shanghai 200233, China. Telephone: +86-021-24058052.
}

( ) Ivyspring International Publisher. Reproduction is permitted for personal, noncommercial use, provided that the article is in whole, unmodified, and properly cited. See http://ivyspring.com/terms for terms and conditions.

Received: 2015.12.26; Accepted: 2016.03.25; Published: 2016.05.25

\begin{abstract}
Bone defects caused by trauma, severe infection, tumor resection and skeletal abnormalities are common osteoporotic conditions and major challenges in orthopedic surgery, and there is still no effective solution to this problem. Consequently, new treatments are needed to develop regeneration procedures without side effects. Exosomes secreted by mesenchymal stem cells (MSCs) derived from human induced pluripotent stem cells (hiPSCs, hiPSC-MSC-Exos) incorporate the advantages of both MSCs and iPSCs with no immunogenicity. However, there are no reports on the application of hiPSC-MSC-Exos to enhance angiogenesis and osteogenesis under osteoporotic conditions. HiPSC-MSC-Exos were isolated and identified before use. The effect of hiPSC-MSC-Exos on the proliferation and osteogenic differentiation of bone marrow MSCs derived from ovariectomized (OVX) rats (rBMSCs-OVX) in vitro were investigated. In vivo, hiPSC-MSC-Exos were implanted into critical size bone defects in ovariectomized rats, and bone regeneration and angiogenesis were examined by microcomputed tomography (micro-CT), sequential fluorescent labeling analysis, microfil perfusion and histological and immunohistochemical analysis. The results in vitro showed that hiPSC-MSC-Exos enhanced cell proliferation and alkaline phosphatase (ALP) activity, and up-regulated mRNA and protein expression of osteoblast-related genes in rBMSCs-OVX. In vivo experiments revealed that hiPSC-MSC-Exos dramatically stimulated bone regeneration and angiogenesis in critical-sized calvarial defects in ovariectomized rats. The effect of hiPSC-MSC-Exos increased with increasing concentration. In this study, we showed that hiPSC-MSC-Exos effectively stimulate the proliferation and osteogenic differentiation of rBMSCs-OVX, with the effect increasing with increasing exosome concentration. Further analysis demonstrated that the application of hiPSC-MSC-Exos $+\beta-T C P$ scaffolds promoted bone regeneration in critical-sized calvarial defects by enhancing angiogenesis and osteogenesis in an ovariectomized rat model.
\end{abstract}

Key words: hiPSC-MSC-Exos, osteoporotic bone defects, osteogenesis, angiogenesis.

\section{Introduction}

As the aging population increases year on year, osteoporosis is becoming one of the most universal and complex skeletal disorders worldwide, especially for postmenopausal women [1]. Osteoporosis is an age-related disease which results from an imbalance between bone formation and resorption so that bone 
resorption is greater than bone formation. Osteoporosis is particularly associated with postmenopausal estrogen deficiency [2, 3]. The disequilibrium of bone remodeling causes microarchitecture deterioration, enhanced bone fragility, and increased risk of fracture [4, 5]. So far, studies have consistently confirmed that bone healing in osteoporotic women and osteoporotic animals is remarkably delayed $[6,7]$. In the clinic, bone defects caused by trauma, severe infection, tumor resection and skeletal abnormalities which occur in osteoporotic patients are major challenges in orthopedic surgery [8]. A variety of currently available strategies are used in treating osteoporotic defects, such as autologous bone transplantation, allogeneic bone transplantation, a combination of scaffold materials with growth factors or cells and so on, but there is still no effective means to solve the problem [9]. Therefore, new treatments are needed to develop novel regeneration procedures and reverse bone loss without side effects.

Recently, MSCs have been extensively investigated in the area of regenerative medicine. MSCs are a heterogeneous subset of stromal stem cells which can be obtained from many adult tissues including human bone marrow, as well as from cord blood, embryonic and fetal membranes, or can be induced by various culture methods [10]. MSCs are easily expanded in culture and are capable of differentiating into osteoblasts, chondrocytes, and adipocytes, which then produce bone, cartilage, and fat tissue as well as other embryonic lineage cells [11, 12]. Nevertheless, the realistic use of MSCs is hampered by their invasive harvesting procedures and ethical and safety hurdles [13].

The increasing concern about stem cell biology has focused attention on the use of reprogrammed cells which are referred as induced pluripotent stem cells (iPSCs) [14]. The attractive feature of iPSCs is their potential for generating patient-specific embryonic stem cells (ESCs) and their unlimited expansion; moreover, they have the ability to differentiate into every organic cell type. IPSCs exhibit the capacity for unlimited growth and are not associated with problems such as immune rejection or the ethical concerns of using ESCs, but they do carry the risk of tumorigenicity [15-18] Consequently mesenchymal stem cells derived from iPSCs (iPSC-MSCs) have been investigated; they incorporate the advantages of both MSCs and iPSCs. Specifically, a high yield of MSCs can be generated from iPSCs, and they are also no longer tumorigenic. iPSC-MSCs have already been used in bone regeneration [19-21].

Recently, growing attention has been paid to exosomes - small (40-100 nm) vesicles of endocytic origin [22-24]. Exosomes are produced by many cell types, and contain various functional proteins, mRNAs, microRNAs, and lipids [25, 26]. Studies have shown that exosomes play a critical role in cell-cell communication [27-29]. Most importantly, exosomes do not contain MHCI or MHCII proteins and overcome all the disadvantages of cell transplantation therapy; researches have showed that their application to xenogeneic animals did not induce overt immune reactions [30-32]. However, to date, there are no reports about the application of hiPSC-MSC-Exos to enhance angiogenesis and osteogenesis under osteoporotic conditions.

The purpose of this study was to investigate the effects of hiPSC-MSC-Exos in promoting bone regeneration through enhanced angiogenesis and osteogenesis in osteoporotic conditions in vivo, and the role of hiPSC-MSC-Exos in stimulating proliferation and differentiation of rBMSCs-OVX into osteoblasts in vitro. Our results show for the first time that hiPSC-MSC-Exos can significantly promote osteogenesis and angiogenesis in critical-sized calvarial bone defects in ovariectomized rats, while in vitro, hiPSC-MSC-Exos can stimulate rBMSCs to proliferate and differentiate into osteoblasts, as well as up-regulating the expression of related osteogenic proteins and genes. hiPSC-MSC-Exos will hopefully become an effective means of treating osteoporotic bone defects in the clinic.

\section{Materials and methods}

This experiment was approved by the Research Ethics Committee at Shanghai Sixth People's Hospital-affiliated Shanghai Jiao Tong University. All animal experiments were conducted in accordance with the Research Ethics Committee of sixth People's Hospital, Shanghai Jiao Tong University, and with the Guide for the Care and Use of Laboratory Animals.

\section{Isolation and identification of hiPSC-MSC-Exos}

The isolation and identification of hiPSC-MSCs-Exos was performed as we have described previously [32, 33]. Briefly, when hiPSC-MSCs reached $80-90 \%$ confluence, the culture medium was replaced by MGro-500 chemically-defined serum-free MSC medium (StemRD), and the cells were cultured for an additional 48 hours. The conditioned medium (CM) from the hiPSC-MSCs was then collected and centrifuged at $300 \times g$ for $10 \mathrm{~min}$ and at 2,000 $\times g$ for 10 min to remove dead cells and cellular debris, respectively. Then, the supernatant was filtered through a Steritop ${ }^{\mathrm{TM}} \quad 0.22 \mu \mathrm{m}$ filter sterilizer (Millipore, Billerica MA, USA). Once more, the supernatant was centrifuged at $4,000 \times g$ to about 200 
$\mu \mathrm{L}$ by ultra-filtration in a $15 \mathrm{~mL}$ Amicon Ultra-15 Centrifugal Filter Unit (Millipore). The ultra-filtered suspension was washed twice with $15 \mathrm{~mL}$ of PBS and re-ultra-filtered at $4,000 \times g$ to $200 \mu \mathrm{L}$. For exosome purification, the suspension was overlaid onto a $30 \%$ sucrose- $\mathrm{D}_{2} \mathrm{O}$ cushion in a sterile Ultra-Clear ${ }^{\mathrm{TM}}$ tube (Beckman Coulter, Brea, CA, USA) and ultra-centrifuged at $100,000 \times g$ for two hours to pellet the small vesicles that correspond to exosomes. The pelleted exosomes were resuspended in $15 \mathrm{~mL}$ of PBS and centrifuged at $4,000 \times \mathrm{g}$ in centrifugal filter units until the final volume was reduced to about $200 \mu \mathrm{L}$. All procedures were performed at $4^{\circ} \mathrm{C}$.

Tunable resistive pulse sensing (TRPS) analysis and western blotting were used to identify hiPSC-MSC-Exos. Briefly, the size distribution and concentration of hiPSC-MSC-Exos were measured using TRPS analysis. TRPS measurements were performed using a qNano platform with an NP100-rated nanopore (Izon Science, Oxford, UK). The membrane was stretched to $45.0 \mathrm{~mm}$. CPC100 particles (Izon Science) were used to calibrate the size and concentration following the manufacturer's instructions. Samples were diluted 1000-fold with $0.22-\mu \mathrm{m}$ filtered PBS and measurement was performed over 3 minutes. Data analysis was carried out using the Izon Control Suite software v2.2 (Izon Science). The presence of exosomal characteristic surface marker proteins including CD9, CD63, and CD81 was analyzed by western blotting.

\section{Animal experiments}

All surgical procedures were conducted under general anesthesia, and postoperative analgesic care was ensured with tramadol. All efforts were made to minimize animal suffering and distress. The animals were anesthetized by intraperitoneal injection of chloral hydrate $(4 \%, 9 \mathrm{~mL} / \mathrm{kg}$ body weight) and all operations were performed under sterile conditions.

\section{Surgical ovariectomy (OVX) and Sham operation (Control)}

Sixty mature female Sprague Dawley (SD) rats (12 weeks old, mean body weight 250-300 g) were used for this study. Animals were randomly divided into OVX and Control groups, $\mathrm{n}=30$ in each.

Osteopenic animal models were established by bilateral ovariectomy as previously described [34]. Briefly, the rats were anesthetized and $10 \mathrm{~mm}$ linear bilateral lumbar lateral skin incisions were created. After exposing muscle and peritoneum by blunt dissection, the bilateral ovaries were gently removed. The same procedure was also performed on all animals of the Sham-operated group, but without removal of the bilateral ovaries. Then, the tissue was repositioned and sutured carefully in layers, and penicillin $(40,000 \mathrm{IU} / \mathrm{mL}, 1 \mathrm{~mL} / \mathrm{kg})$ was administered by injection for 3 days. Two months after creation of the OVX model, distal femurs were harvested to confirm the development of osteoporosis by micro-CT analysis.

\section{Preparation of rBMSCs-OVX}

The isolation and identification of rBMSCs-OVX were conducted as previously described [35]. Briefly, bone marrow was harvested from the femora and tibiae of SD rats. The cells from one rat were seeded onto $10 \mathrm{~cm}^{2}$ plastic tissue culture plates in minimum essential medium alpha (MEM-a; Invitrogen, Carlsbad, CA, USA) supplemented with $10 \%$ fetal bovine serum, $100 \mathrm{IU} / \mathrm{mL}$ penicillin and $100 \mu \mathrm{g} / \mathrm{mL}$ streptomycin, then incubated at $37^{\circ} \mathrm{C}$ in an incubator containing $5 \% \quad \mathrm{CO}_{2}$ for $48 \mathrm{~h}$. Subsequently, the non-adherent cells were discarded while the adherent cells were allowed to grow to $80 \%$ confluence and these were defined as passage one cells (P1). P3 cells were used for all experiments.

\section{Proliferation assay}

The effect of hiPSC-MSC-Exos on the proliferation of rBMSCs-OVX was evaluated using a cell counting kit-8 (CCK-8; Dojindo) according to the manufacturer's instructions. The cells were randomly divided into three groups and treated as follows: control: the same amount of cell culture medium was added; $100 \mu \mathrm{g} / \mathrm{mL}$ Exos: hiPSC-MSC-Exos were added to a concentration of $100 \mu \mathrm{g} / \mathrm{mL} ; 200 \mu \mathrm{g} / \mathrm{mL}$ Exos: hiPSC-MSC-Exos were added to a concentration of $200 \mu \mathrm{g} / \mathrm{mL}$. Briefly, $3 \times 10^{3}$ rBMSCs-OVX (p3) were plated into 96-well plates and allowed to proliferate for $48 \mathrm{~h}$, then exosomes were added to the wells and cultured for a further 1, 3 or 5 days. At the end of these incubation periods, cells were incubated with 10 $\mu \mathrm{L}$ CCK8 solution for approximately $3 \mathrm{~h}$. Cell proliferation was calculated from the absorbance determined at $450 \mathrm{~nm}$ using a micro-plate reader.

\section{ALP activity assay and alizarin red $S$ staining}

Three groups of cells were plated into 6-well plates and cultured in fresh osteogenic differentiation medium (OM) consisting of $50 \mu \mathrm{M}$ ascorbate-2-phosphate, $10 \mathrm{mM} \beta$-glycero-phosphate, and $100 \mathrm{nM}$ dexamethasone and containing different treatments for 14 days. The cells were then lysed and ALP activity was assayed with an ALP staining kit according to the manufacturer's protocol (Beyotime Institute of Biotechnology, Shanghai, China). Bone mineralization was determined by alizarin red $\mathrm{S}$ (Sigma, St Louis, MO, USA) staining. Cells were cultured with $2 \%$ alizarin red at $\mathrm{pH} 4.2$ for $10 \mathrm{~min}$ and then washed using distilled water. Mineralized 
nodules were examined by phase-contrast microscopy at 21 days.

\section{Real-time quantitative polymerase chain reaction ( $R T$-qPCR)}

Total RNA was isolated from cells in the three groups using the Trizol method (Invitrogen) at 3 and 7 days. Messenger RNA was reverse-transcribed to cDNA and amplified using a One Step SYBR RT-PCR Kit (TaKaRa Bio, Shiga, Japan). The cDNA was then amplified using SYBR Premix Ex Taq (Takara) in combination with a ViiA7 Real-Time PCR System (ABI, Life Technology, Carlsbad, CA, USA). $\beta$-actin was used as an internal control for PCR amplification; all samples were tested in triplicate. Primer information is listed in Table 1.

Table 1. Sequences of primers used in RT-qPCR.

\begin{tabular}{ll}
\hline Molecule & Primer sequences \\
\hline ALP & GTTTTCTGTTCTGTAAGACGGG \\
& GCCGTTAATTGACGTTCCGA \\
RUNX-2 & CCGAGCTACGAAATGCCTCT \\
& GGACCGTCCACTGTCACTTT \\
COL1-A1 & ACATGTTCAGCTTTGTGGACC \\
& AGGTTCCACGTCTCACCAT \\
\hline
\end{tabular}

\section{Western blotting}

After 7 days, the cultured rBMSCs were lysed in RIPA buffer (Bio-Rad, Hercules, CA, USA) to extract proteins to detect the expression of bone-related proteins such as Osteopontin (OPN), Runt-related transcription factor 2 (RUNX-2) and Collagen type I (COL1). Proteins were separated by electrophoresis on an $8 \%$ SDS-PAGE gel, then transferred to a PVDF membrane (Millipore). The membrane was blocked with 5\% non-fat milk prior to incubating with each primary antibody (abcam, 1:1000 dilution), followed by the respective secondary antibody (abcam, 1:5000 dilution). The membrane was washed with TBST after each incubation, then washed and reacted with a chemi-luminescent substrate (Thermo Scientific, Waltham, MA, USA). The bands were imaged. This experiment was repeated in triplicate.

\section{Surgical craniotomies-establishment of critical size defects}

In the in vivo study, classical porous $\beta$-TCP scaffolds (5 $\mathrm{mm}$ diameter and $2 \mathrm{~mm}$ depth) with an average pore size of $500 \mu \mathrm{m}$ and $75 \%$ porosity were used as exosome carriers. Aliquots of 100 or $200 \mu \mathrm{g}$ hiPSC-MSC-Exos were dropped onto each $\beta$-TCP scaffold under sterile conditions and lyophilized for at least $4 \mathrm{~h}$.

Surgical procedures were carried out as previously described [36]. Briefly, 27 osteopenic animals were anesthetized by intraperitoneal injection, a $1.5-\mathrm{cm}$ sagittal incision was made in the scalp, and the calvarium was exposed by blunt dissection. Two bilateral $5 \mathrm{~mm}$ diameter critical-sized calvarial defects were created using a dental trephine, and then scaffolds were implanted into the defects. The rats were then randomly divided into three groups: $\beta$-TCP group: $\beta$-TCP only (9), $\beta$-TCP+100 $\mu \mathrm{g}$ Exos group: $100 \mu \mathrm{g}$ hiPSC-MSC-Exos $+\beta$-TCP (9), $\beta-\mathrm{TCP}+200 \mu \mathrm{g} \quad$ Exos group: $200 \mu \mathrm{g}$ hiPSC-MSC-Exos $+\beta$-TCP (9). Eight weeks after surgery, the rats were sacrificed and crania were harvested and fixed in a $4 \%$ paraformaldehyde solution buffered with $0.1 \mathrm{M}$ phosphate solution $(\mathrm{pH}$ 7.2) before further analysis.

\section{Micro-CT analysis}

All the harvested specimens were examined using a micro-CT system (mCT-80, Scanco Medical, Brüttisellen, Switzerland) (1) to identify the establishment of the osteoporotic model with microarchitectural changes, and (2) to evaluate new bone formation within the defect region. Briefly, the specimens were scanned with a thickness of $0.018 \mathrm{~mm}$ per slice in medium-resolution mode, with a 1,024 reconstruction matrix, and $200 \mathrm{~ms}$ integration time. After 3D reconstruction, bone mineral density (BMD), bone volume fraction $(\mathrm{BV} / \mathrm{TV})$, trabecular number (Tb.N), trabecular thickness (Tb.Th) and trabecular separation (Tb.Sp) were automatically determined to confirm the osteoporotic model, while the BMD and $\mathrm{BV} / \mathrm{TV}$ values in the defect regions were used to evaluate new bone formation using the auxiliary software (Scanco) [37].

\section{Sequential fluorescent labeling}

At 2, 4 and 6 weeks after the craniotomies, the rats were intraperitoneally injected with tetracycline (TE, $25 \mathrm{mg} / \mathrm{kg}$ of body weight), alizarin red (AL, 30 $\mathrm{mg} / \mathrm{kg}$ of body weight) and calcein (CA, $20 \mathrm{mg} / \mathrm{kg}$ of body weight), and the mineralized tissue was observed by the trichromatic sequential fluorescent labeling method [37]. The images in yellow represent $\mathrm{TE}$, the images in red represent $\mathrm{AL}$, and the images in green represent $\mathrm{CA}$ indicating bone formation and mineralization at 2, 4 and 6 weeks after operation, respectively. The percentage of $\mathrm{TE}, \mathrm{AL}$ and $\mathrm{CA}$ staining for each group assessed at week 8 after implantation by histomorphometric analysis.

\section{Microfil perfusion}

To compare blood vessel formation in vivo, rats were perfused with Microfil (Flowtech, Carver, MA, USA) after euthanasia. In brief, the rib cage was opened, the descending aorta was clamped, and an 
angiocatheter was used to penetrate the left ventricle. After the inferior vena cava was incised, $20 \mathrm{~mL}$ heparinized saline was perfused, then $20 \mathrm{~mL}$ of Microfil was perfused at $2 \mathrm{~mL} / \mathrm{min}$. Finally, the rats were kept overnight at $4^{\circ} \mathrm{C}$ [38]. The specimens were decalcified in decalcifying solution for about two weeks, then scanned with a thickness of $0.009 \mathrm{~mm}$ per slice in medium-resolution mode, with a 1,024 reconstruction matrix, and $200 \mathrm{~ms}$ integration time. New blood vessel formation in the calvarial defects was presented as 3-D reconstructed images; morphometric analysis was performed to evaluate local blood vessel area and blood vessel number in the bone defects.

\section{Histological and immunohistochemical analysis}

One part of each cranium was dehydrated through ascending concentrations of alcohol from $70 \%$ to $100 \%$, and then embedded in polymethylmethacrylate (PMMA). After hardening, the longitudinal sections of the crania were cut into 150-200 $\mu \mathrm{m}$ thick slices using a microtome (Leica Microsystems, Wetzlar, Germany), and then glued onto a plastic support and polished to a final thickness of approximately $50 \mu \mathrm{m}$. First, the sections were examined by confocal laser scanning microscopy (CLSM) (Leica) for fluorescent labeling, then new bone formation and mineralization was quantified at four locations. The mean value of the four measurements was calculated to assess average values of each group. Next, the sections were stained with van Gieson's picrofuchsin to evaluate new bone formation, with red areas representing new bone

A

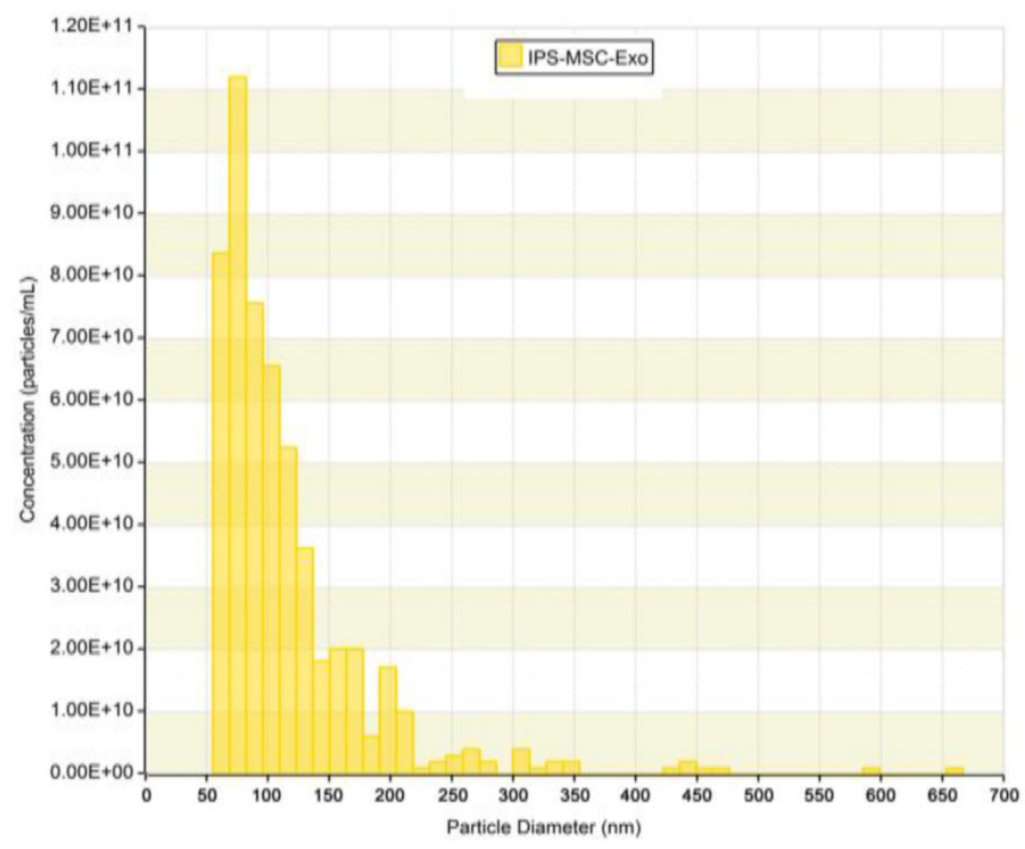

formation, and black areas representing $\beta$-TCP [37]. The area of new bone formation was evaluated quantitatively at four randomly-selected sections by Image Pro 5.0 (Media Cybernetics, Rockville, MD, USA).

The other part of each cranium was decalcified in decalcifying solution for about two weeks, dehydrated through gradient alcohols, embedded in paraffin, and cut into $5 \mu \mathrm{m}$ thick sections. Immunohistochemical staining was performed for osteocalcin (OCN) and osteopontin (OPN) to evaluate osteogenesis, while CD31 immunohistochemistry was used to detect angiogenesis in specimens.

\section{Statistical analysis}

All the above data were analyzed as mean \pm standard deviation (SD). Differences between groups were calculated by one-way analysis of variance (ANOVA). Statistical analysis was conducted using SPSS 17.0 software (IBM Corp. Armonk, NY, USA). The difference was significant when $P$ values $<0.05$.

\section{Results}

\section{Characterization of exosomes}

TRPS analysis and western blotting were used to identify the hiPSC-MSC-Exos. The results showed that the large majority of these particles exhibited a cup- or round-shaped morphology with a size ranging from 50 to $150 \mathrm{~nm}$ (Fig. 1A), indicating that they were exosomes. Moreover, the results of western blotting showed these particles expressed the characteristic surface markers, such as CD9, CD63 and CD81 (Fig. 1B), which further confirmed them to be exosomes.
Figure 1. Characterization of hiPSC-MSC-Exos. (A) Particle size distributions and concentration of hiPSC-MSC-Exos measured by TRPS. (B) Western blot analysis of the exosomal surface markers CD9, CD63 and CD81 in hiPSC-MSC-Exos.
B

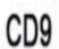

CD63

CD81 

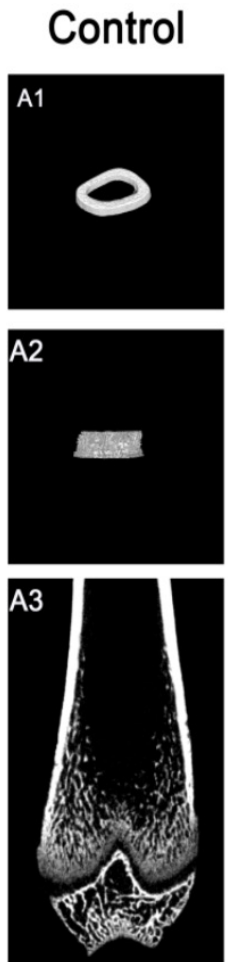

ovX
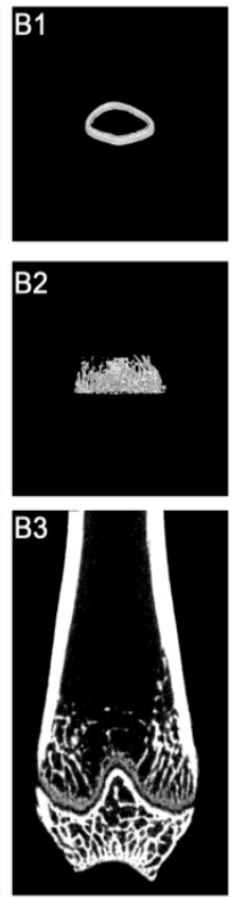

C

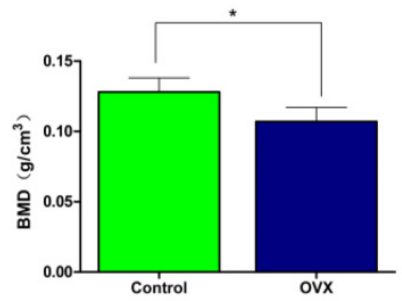

E

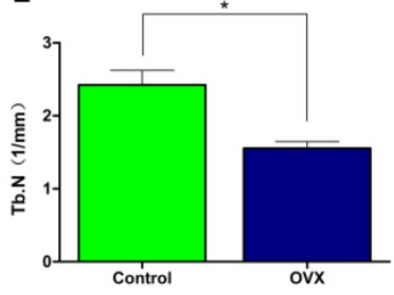

D

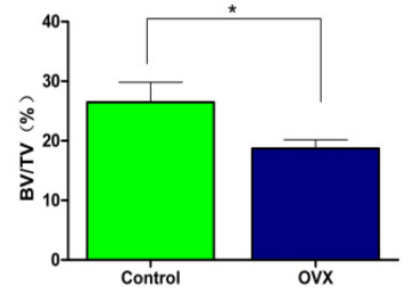

$\mathrm{F}$

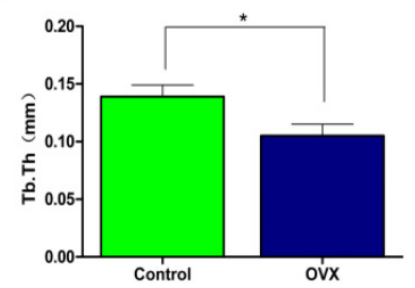

G

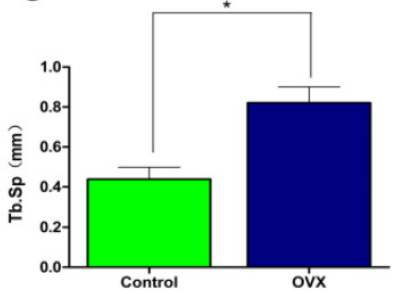

Figure 2. Establishment of the OVX model. 3D-MicroCT images of the distal femur in Control groups (A1-A3) and OVX groups (B1-B3). Quantitative data of BMD (C), BV/TV (D), Tb.N (E), Tb.Th (F), Tb.Sp (G) in the distal femur ( $n=6$ per group). $* P<0.05$.

\section{Confirmation of the osteoporotic model}

Micro-CT 3D images of the distal femur revealed that OVX rats (Fig. 2B) had thinner trabecular bone and disorganized trabecular architecture, with decreased interconnectivity and increased separation as well as thinning of the cortical bones compared to the control rats (Fig.2A). Quantification of the microstructural parameters revealed that the BV/TV $(18.716 \pm 1.46 \%)$, Tb.N $(1.557 \pm 0.09 / \mathrm{mm})$, Tb.Th $(0.105$ $\pm 0.01 \mathrm{~mm})$, and BMD $\left(0.107 \pm 0.01 \mathrm{~g} / \mathrm{cm}^{3}\right)$ in the distal femur were significantly lower in OVX rats compared to those in control rats: BV/TV $(26.499 \pm 3.32 \%), \mathrm{Tb} . \mathrm{N}$ $(2.424 \pm 0.20 / \mathrm{mm}), \mathrm{Tb} . \mathrm{Th}(0.139 \pm 0.01 \mathrm{~mm})$, and BMD $\left(0.128 \pm 0.01 \mathrm{~g} / \mathrm{cm}^{3}\right)(P<0.05)$ (Fig. $\left.2 \mathrm{C}-\mathrm{F}\right)$, while the Tb.Sp $(0.821 \pm 0.08 \mathrm{~mm})$ was significantly higher in OVX rats compared to control rats Tb.Sp $(0.439 \pm 0.06$ mm) $(P<0.05)$ (Fig. 2G). The above results showed that there was significantly difference in OVX rats compared to control rats, which confirmed the establishment of osteoporotic rats models.

\section{hiPSC-MSC-Exos promoted osteoblast proliferation, differentiation and bone formation.}

The effect of hiPSC-MSC-Exos on the proliferation of rBMSCs-OVX was evaluated by the CCK-8 method (Fig. 3D). The results revealed that there was a significant difference between the 200 $\mu \mathrm{g} / \mathrm{mL}$ Exos groups and the control group, and also with the $100 \mu \mathrm{g} / \mathrm{mL}$ Exos group $(\mathrm{P}<0.05)$. It showed that hiPSC-MSC-Exos promoted proliferation of rBMSCs-OVX and increased with increasing concentrations of exosomes. The hiPSC-MSC-Exos were added to rBMSCs-OVX 6-well plates in the presence of OM to quantify ALP activity as an early marker of osteogenic differentiation. As shown in Fig. 3A1-A3, more intensive ALP staining was observed in cells treated with $200 \mu \mathrm{g} / \mathrm{mL}$ Exos compared with others. Moreover, quantitative analysis of ALP activity (Fig. 3C) showed that there was a significant difference between the $200 \mu \mathrm{g} / \mathrm{mL}$ Exos group and the Control and $100 \mu \mathrm{g} / \mathrm{mL}$ Exos groups $(\mathrm{P}<0.05)$ at 7 days. The results of alizarin red $S$ staining showed that the deposition of mineral was markedly enhanced compared with the Control group (Fig. 3B1-B3). The results of ALP staining and alizarin red $S$ staining showed that hiPSC-MSC-Exos promoted osteoblastic differentiation of rBMSCs-OVX.

The RT-qPCR assay results showed enhanced mRNA expression of RUNX-2, COL1 and ALP in cells 
treated with hiPSC-MSC-Exos, with significant differences compared with control groups $(P<0.05)$, while there was also a significant difference between the $200 \mu \mathrm{g} / \mathrm{mL}$ and $100 \mu \mathrm{g} / \mathrm{mL}$ Exos groups $(P<0.05)$. The results showed that hiPSC-MSC-Exos up-regulated the expression of osteogenesis-related genes in osteogenic induction medium (Fig. 3F-H).

The expression levels of bone-related proteins were also analyzed by western blot. As shown in Fig. $3 \mathrm{E}$, the protein expression levels of OPN, RUNX-2, and COL1 in cultures treated with hiPSC-MSC-Exos were remarkably up-regulated compared with the control group $(P<0.05)$ at 3 and 7 days, and there was a significant difference between the $200 \mu \mathrm{g} / \mathrm{mL}$ Exos groups and the $100 \mu \mathrm{g} / \mathrm{mL}$ Exos group $(P<0.05)$. The results showed that hiPSC-MSC-Exos remarkably enhanced the expression of bone-related proteins by rBMSCs-OVX.

The in vitro researches demonstrated that hiPSC-MSC-Exos effectively stimulated the proliferation and enhanced osteogenic differentiation

Control
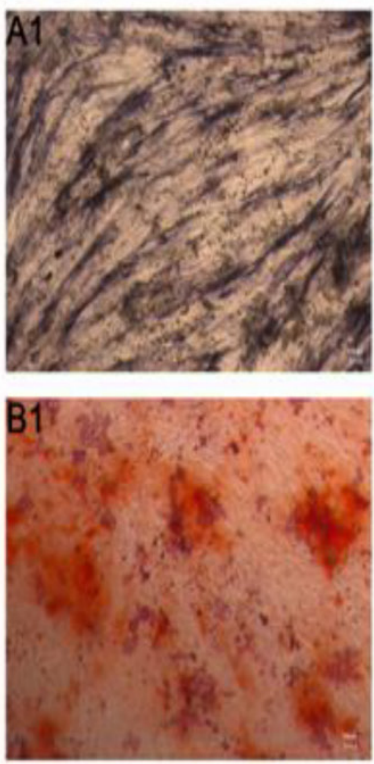

100ug/ml Exos
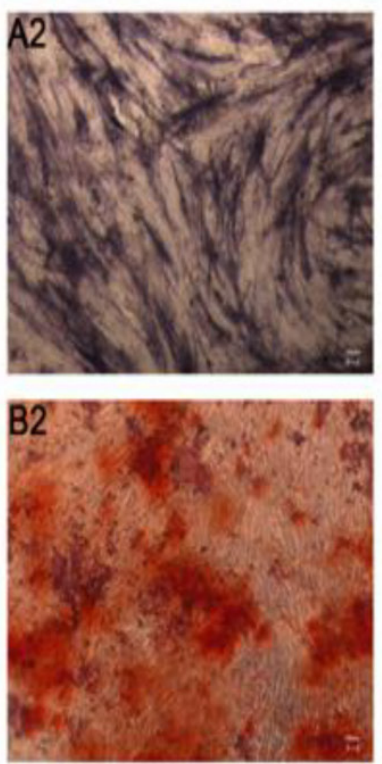

of rBMSCs-OVX, with the effect increasing with increasing exosome concentration.

\section{Micro-CT analysis of bone regeneration in calvarial defects}

Three-dimensional micro-CT reconstruction images revealed the morphology of the newly-formed bone (Fig. 4A1-C1, Fig.4A2-C2). In the sagittal view (Fig. 4A3-C3), more newly-formed bone was observed in the $\beta-\mathrm{TCP}+200 \mu \mathrm{g}$ Exos group than in the $\beta$-TCP+100 $\mu \mathrm{g}$ Exos group or the $\beta$-TCP alone group. Quantitative analysis of the newly-formed bone was performed using the analysis system. The results showed that the local BMD was markedly higher in the $\beta$-TCP+200 $\mu \mathrm{g}$ Exos group $\left(0.372 \pm 0.054 \mathrm{~g} / \mathrm{cm}^{3}\right)$ than in the $\beta-\mathrm{TCP}+100 \mu \mathrm{g}$ Exos group $(0.181 \pm$ $\left.0.037 \mathrm{~g} / \mathrm{cm}^{3}\right)$ or the $\beta$-TCP group $\left(0.05 \pm 0.013 \mathrm{~g} / \mathrm{cm}^{3}\right)$ $(P<0.05)$ (Fig.4D). Moreover, analysis of $\mathrm{BV} / \mathrm{TV}$ values showed the same differences as the BMD data (Fig. 4E). The results indicated that hiPSC-MSC-Exos can improve bone regeneration under osteoporotic conditions, and that there is a dose-effect relationship.

\section{0ug/ml Exos}
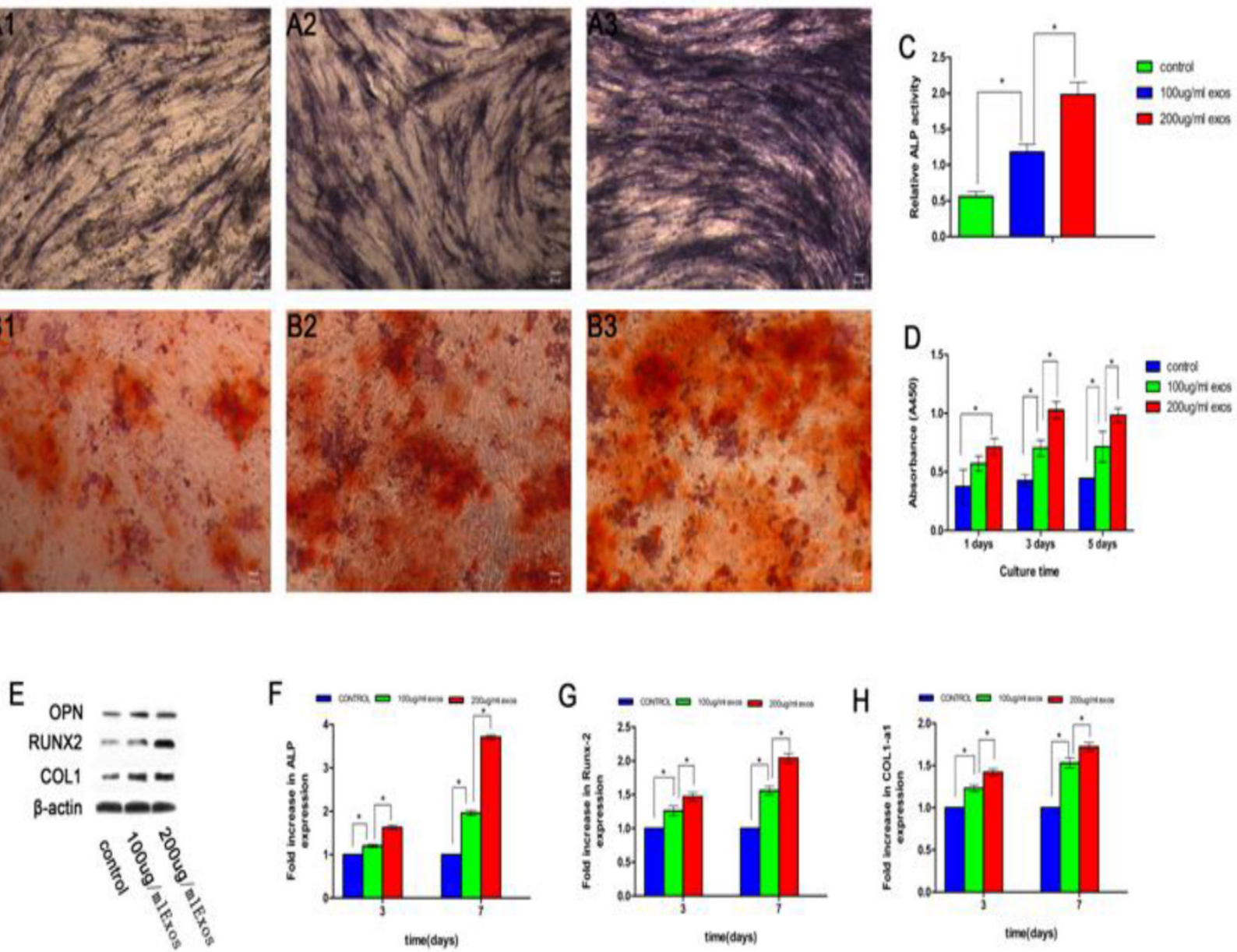

Figure 3. hiPSC-MSC-Exos promoted osteoblast proliferation, differentiation and bone formation. ALP staining at 14 days (A1-A3), alizarin red S staining at 21 days (B1-B3) and (C) quantitative analysis of ALP activity were used to evaluate the effect of hiPSC-MSC-Exos on osteogenic differentiation of rBMSCs-OVX. (D) The effect of hiPSC-MSC-Exos on the proliferation of rBMSCs-OVX was evaluated by the CCK-8 method. (E) The protein levels of OPN, RUNX-2 and COL1 measured by western blotting. The mRNA expression levels of ALP (F), RUNX-2 (G) and COL1 (H) were measured by qRT-PCR, at 3 days and 7 days. (*indicates significant differences, $P<0.05$; Scale bar: $100 \mu m)$. 

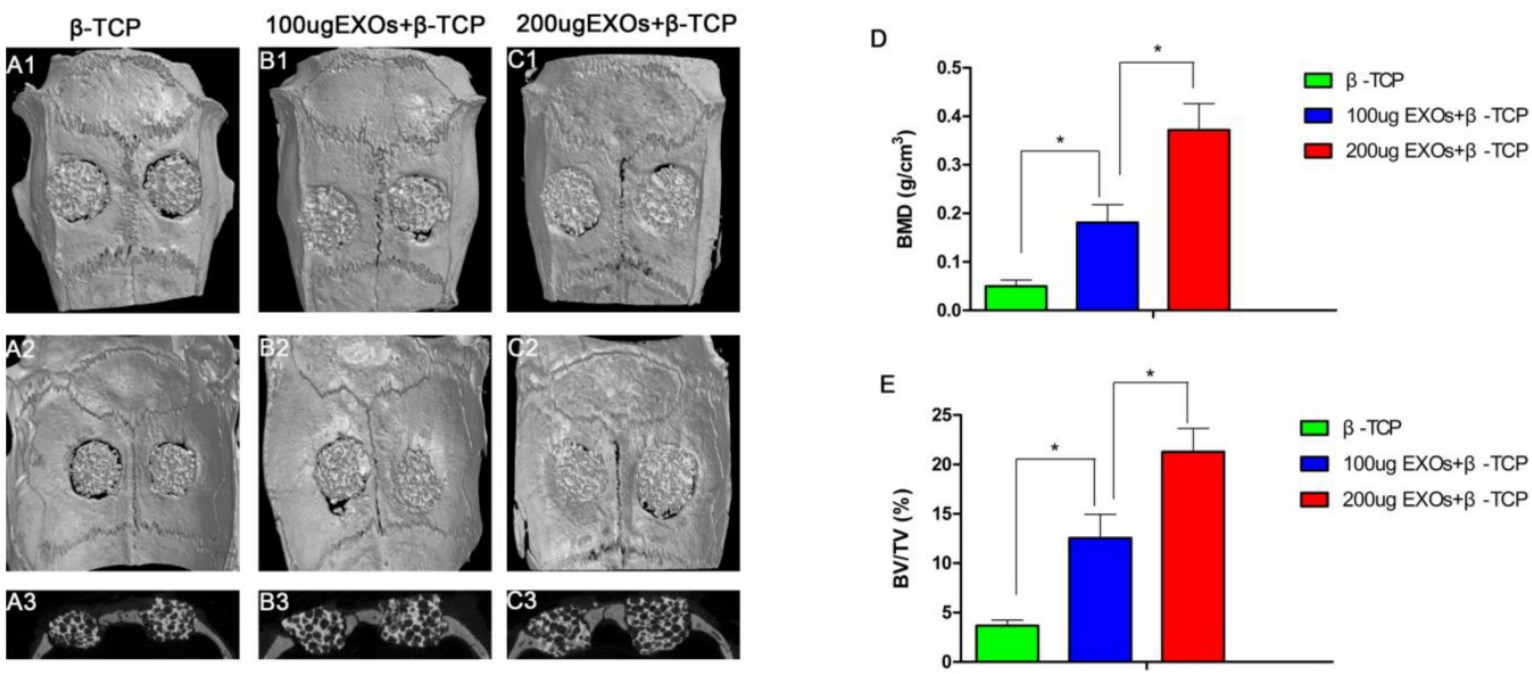

Figure 4. The analysis of hiPSC-MSC-Exos+ $\beta$-TCP scaffolds promoted bone regeneration by Micro-CT. Representative 3D reconstruction of superficial (A1-C1), interior images (A2-C2) and sagittal images (A3-C3) of calvarial bone defects taken at 8 weeks after implantation of hiPSC-MSC-Exos and scaffolds; (D) Morphometric analysis of bone mineral density (BMD) and (E) bone volume/total volume (BV/TV) by Micro-CT for each group at 8 weeks post operation $(*$ indicates significant differences, $P<0.05)$.
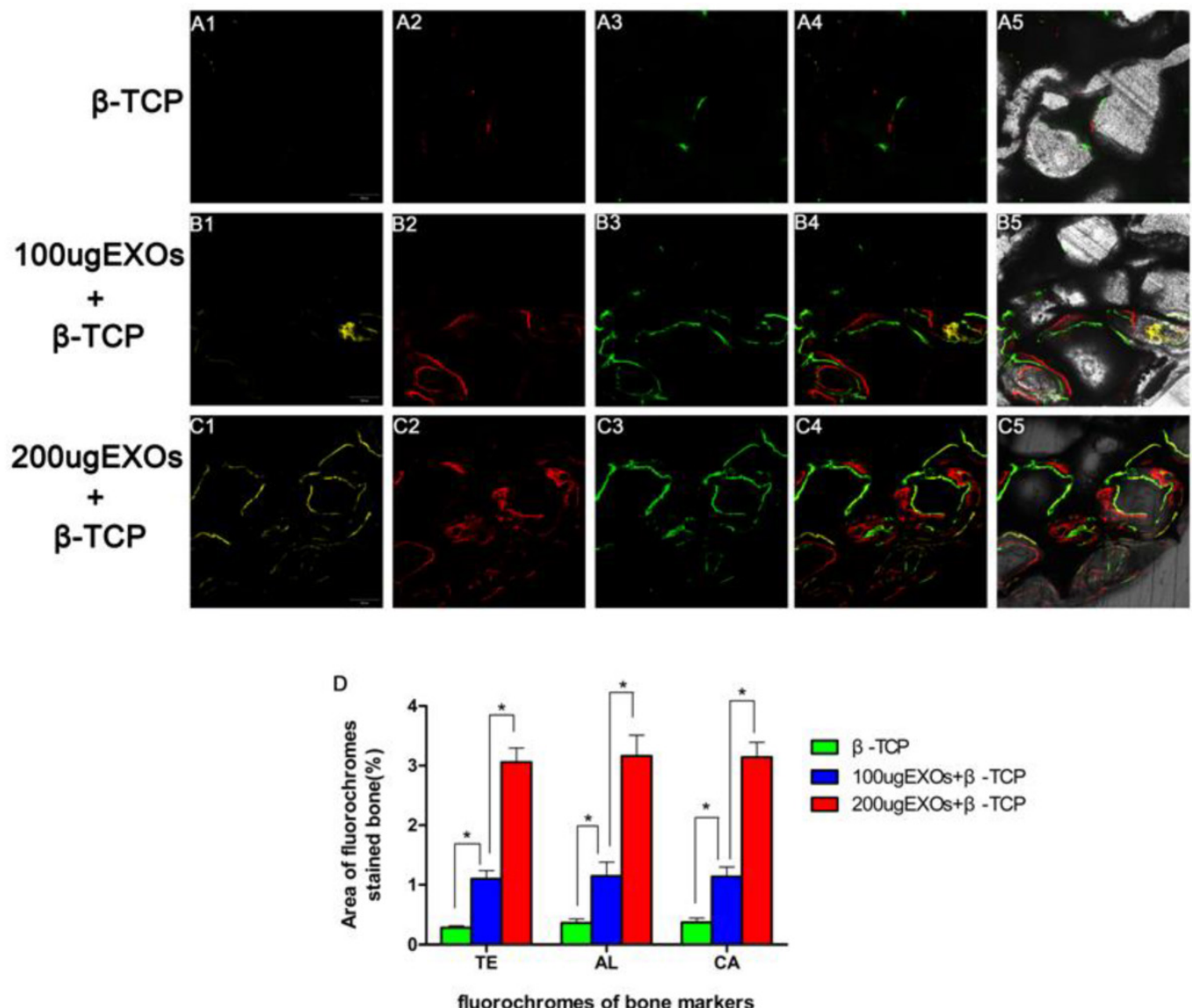

Figure 5. The analysis of hiPSC-MSC-Exos $+\beta-T C P$ scaffolds promoted bone regeneration by sequential fluorescent labeling. The images $(A 1-C 1)$ in yellow represent TE, the images (A2-C2) in red represent $A L$, and the images (A3-C3) in green represent $C A$ indicating bone formation and mineralization at 2, 4 and 6 weeks after operation, respectively. (A4-C4): merged images of the three fluorochromes, (A5-C5): merged images of the three fluorochromes together with a bright field confocal laser microscope image; (D) The percentage of TE, AL and CA staining for each group assessed at week 8 after implantation by histomorphometric analysis. $\left({ }^{*}\right.$ indicates significant differences, $P<$ 0.05 ; Scale bar: $200 \mu \mathrm{m})$.

\section{Fluorochrome labeling histomorphometric analysis}

As revealed in Fig. 5, new bone formation and mineralization were analyzed at 2, 4 and 6 weeks by fluorescence labeling. At 2 weeks, the percentage of TE labeling (yellow) in the $\beta$-TCP $+200 \mu \mathrm{g}$ Exos group $(3.06 \pm 0.236 \%)$ was larger than that in the $\beta$-TCP group $(0.28 \pm 0.035 \%)$ or the $\beta$-TCP+100 $\mu \mathrm{g}$ Exos 
group $(1.1 \pm 0.139 \%)(P<0.05)$, but there was also a significant difference between the $\beta$-TCP+100 $\mu$ g Exos group and the $\beta$-TCP group $(P<0.05)$ (Fig. 4A1-C1). At 4 weeks, the highest percentage of AL labeling (red) was observed in the $\beta-\mathrm{TCP}+200 \mu \mathrm{g}$ Exos group $(3.16 \pm 0.351 \%)$, but there was also a significant difference between the $\beta$-TCP $+100 \mu \mathrm{g}$ Exos group $(1.15 \pm 0.231 \%)$ and the $\beta$-TCP groups $(0.36 \pm 0.069 \%)$ $(P<0.05)$ (Fig. 5A2-C2). At 6 weeks, the percentage of CA labeling (green) in the $\beta-\mathrm{TCP}+200 \mu \mathrm{g}$ Exos group $(3.14 \pm 0.25 \%)$ was significantly higher than that in the $\beta$-TCP group $(0.37 \pm 0.073 \%)$ or the $\beta$-TCP $+100 \mu \mathrm{g}$ Exos group $(1.14 \pm 0.159 \%)(P<0.05)$, but there was also a significant difference between the $\beta$-TCP+100 $\mu \mathrm{g}$ Exos group and the $\beta$-TCP group $(\mathrm{P}<0.05)$ (Fig. $5 \mathrm{~A} 3-\mathrm{C} 3)$. The results found that $\beta$-TCP scaffolds $+200 \mu \mathrm{g}$ hiPSC-MSC-Exos could more effectively improve bone regeneration in osteoporotic rats.

\section{Neovascularization of defects}

Three-dimensional micro-CT images showed that new angiogenesis in the $\beta-\mathrm{TCP}+200 \mu \mathrm{g}$ Exos group and the $\beta$-TCP+100 $\mu \mathrm{g}$ Exos group was markedly increased compared with the $\beta$-TCP group, and the $\beta-\mathrm{TCP}+200 \mu \mathrm{g}$ Exos group also showed higher neovascularization than the $\beta-\mathrm{TCP}+100 \mu \mathrm{g}$ Exos group (Fig.6A1-A3). Quantification of the new blood vessel area (Fig. 6B) and vessel number (Fig.6C) verified the above results. There were significant differences between the $\beta$-TCP+200 $\mu$ g Exos group $(67.17 \pm 3.76 \%)$, the $\beta$-TCP $+100 \mu \mathrm{g}$ Exos group (30.5 \pm $4.14 \%)$ and the $\beta$-TCP group $(4.33 \pm 1.97 \%)$ in the number of newly-formed vessels $(\mathrm{P}<0.05)$, but the $\beta-\mathrm{TCP}+200 \mu \mathrm{g}$ Exos group showed the greatest area of neovascularization. The results indicated that $\beta$-TCP scaffolds $+200 \mu \mathrm{g}$ hiPSC-MSC-Exos could more markedly enhanced new angiogenesis in the area of bone defects.

\section{Histological and immunohistochemical analysis}

The results of Van Gieson staining of undecalcified specimens showed that bone regeneration was markedly increased in the $\beta-\mathrm{TCP}+200 \mu \mathrm{g}$ Exos group $(40.11 \pm 2.52 \%)$, the percentage of new bone formation area was significantly greater than that in the $\beta$-TCP group $(4.52 \pm 1.2 \%)$ or the $\beta$-TCP $+100 \mu \mathrm{g}$ Exos group $(20.45 \pm$ $2.45 \%)(\mathrm{P}<0.05)$, and there was also a significant difference between the $\beta$-TCP+100 $\mu \mathrm{g}$ Exos group and the $\beta$-TCP group $(\mathrm{P}<0.05)$ (Fig.7). The resulte showed the same trend as the results of Micro-CT analysis of bone regeneration.

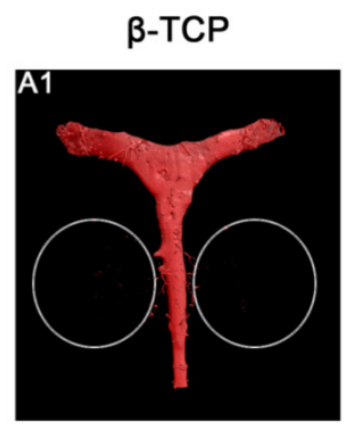

100ugEXOs+ $\beta-$ TCP 200ugEXOs+ $\beta-\mathrm{TCP}$
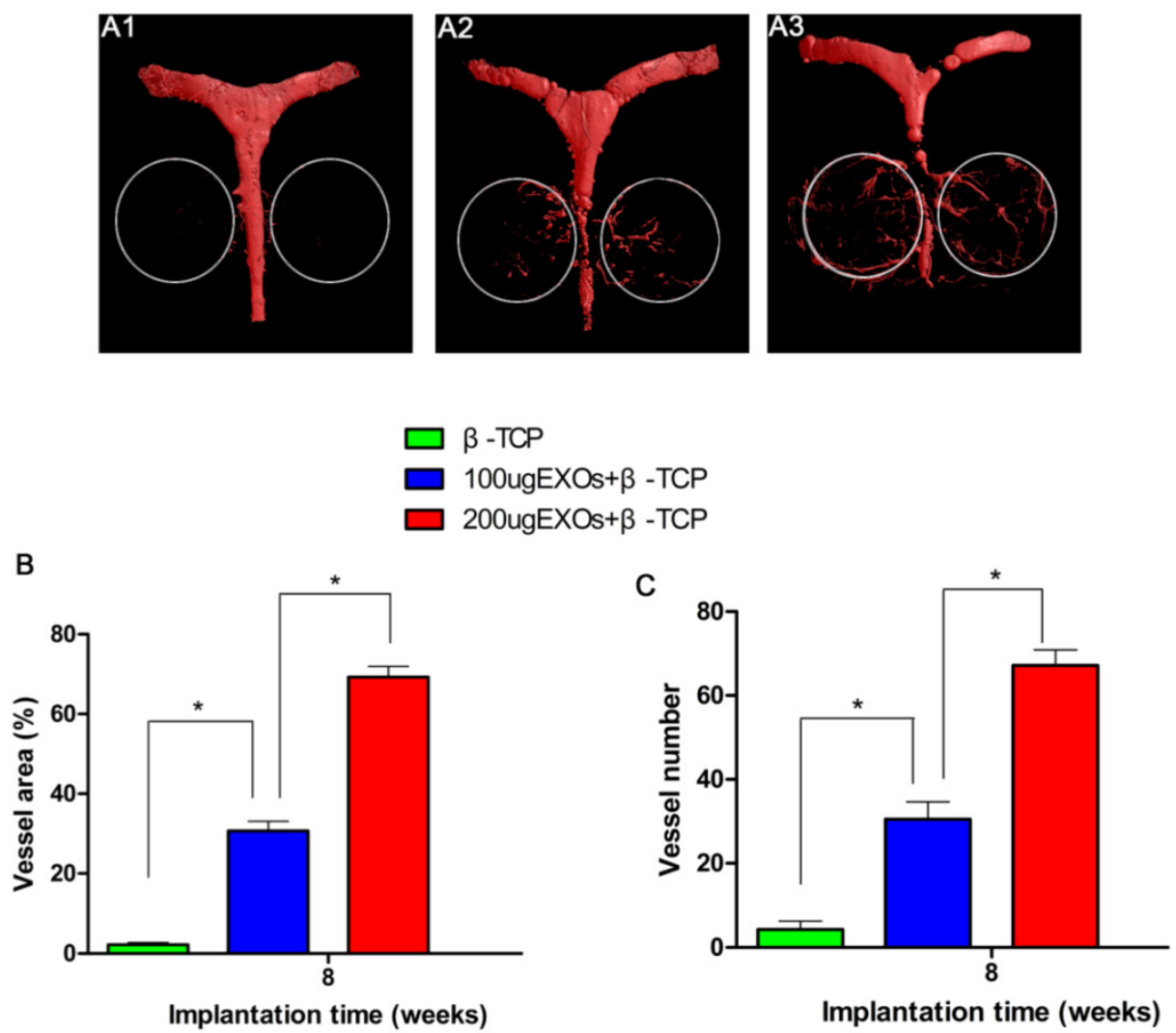

Figure 6. The analysis of hiPSC-MSC-Exos $+\beta-T C P$ scaffolds promoted angiogenesis by Microfil perfusion. New blood vessel formation in the calvarial defects is presented as $3-D$ reconstructed images (A1-A3). Morphometric analysis was performed to evaluate local blood vessel area $(B)$, and blood vessel number $(C)$ in the bone defects $(* P<0.05)$. 

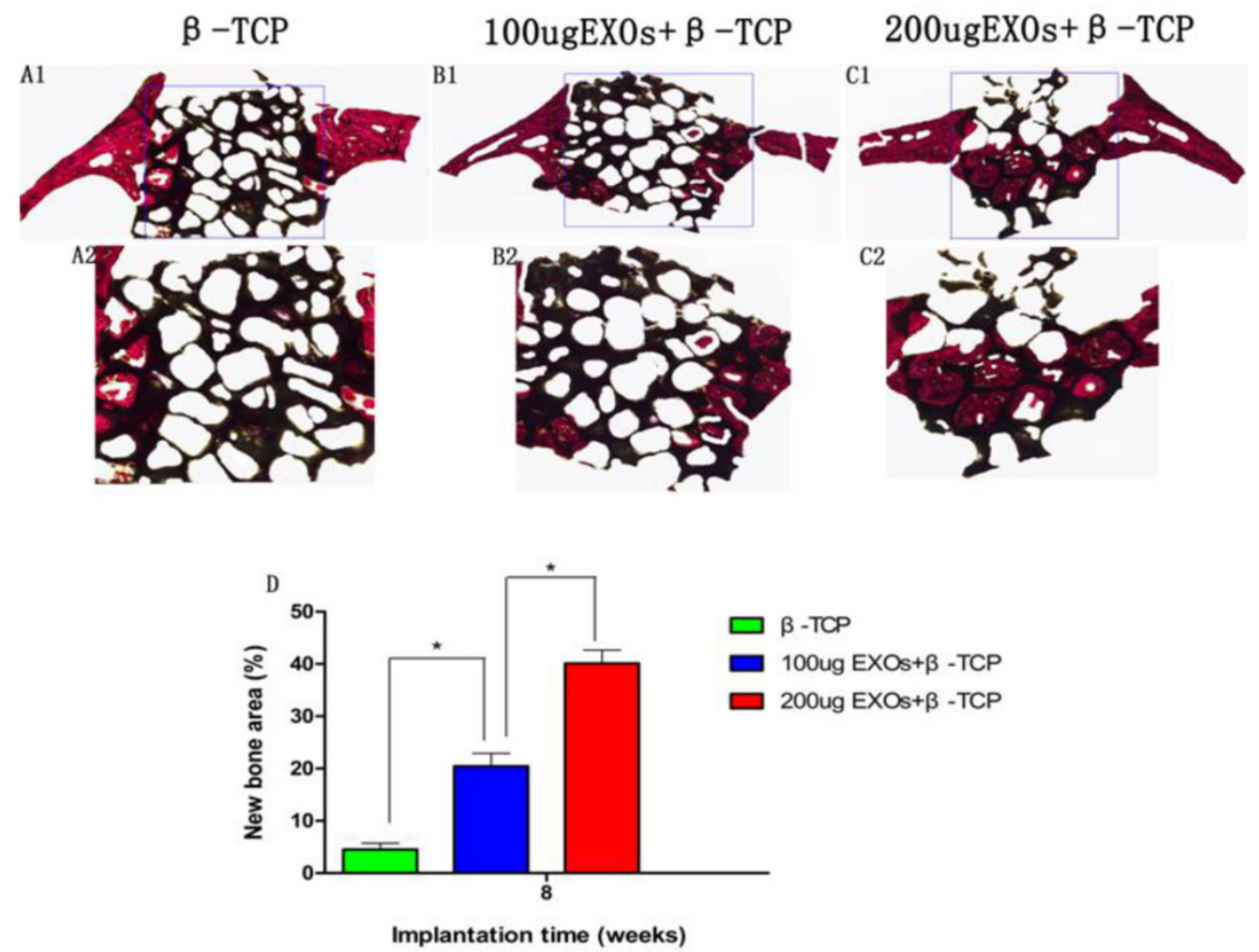

Figure 7. The histological analysis of hiPSC-MSC-Exos+ $\beta$-TCP scaffolds promoted bone regeneration. The un-decalcified crania were sectioned and stained with van Gieson's picrofuchsin $(\mathrm{Al}-\mathrm{Cl})$. The new bone area and $\beta$-TCP residue are shown in red and black, respectively. (D) The percentage of new bone area was assessed at 8 weeks after implantation by histomorphometric analysis $(* P<0.05)$.

The osteogenic markers OCN and OPN and the angiogenic marker CD31 were detected by immunohistochemical staining of decalcified crania (Fig. 8), with the results showing that there was virtually no obvious positive staining for OCN (Fig. 7A1) or OPN (Fig. 8B1) in the $\beta$-TCP group, but that positive brown staining for OCN and OPN were apparent in the $\beta-\mathrm{TCP}+100 \mu \mathrm{g}$ Exos group (Fig. 8A2, B2) and $\beta-\mathrm{TCP}+200 \mu \mathrm{g}$ Exos group (Fig. 8A3, B3). However, the most obvious positive staining for $\mathrm{OCN}$ and OPN was found in the $\beta-\mathrm{TCP}+200 \mu \mathrm{g}$ Exos group. CD31-positive brown staining was more obvious in the $\beta$-TCP+100 $\mu \mathrm{g}$ Exos group (Fig. 8C2) or the $\beta-\mathrm{TCP}+200 \mu \mathrm{g}$ Exos group (Fig. 8C3) compared with the $\beta$-TCP group (Fig. $8 \mathrm{C} 1$ ), and the most obvious positive staining was observed in the $\beta-\mathrm{TCP}+200 \mu \mathrm{g}$ Exos group. The results indicated that $\beta$-TCP scaffolds+200 $\mu \mathrm{g}$ hiPSC-MSC-Exos could more markedly promoted the expression of osteogenic and angiogenic markers in the area of bone defects.

\section{Discussion}

Osteoporotic defects are common clinical complications of postmenopausal osteoporosis. Currently, the prevention and therapeutic approaches for osteoporosis are mainly focused on medical treatment, including bisphosphonates, calcitonin, and selective estrogen receptor modulators [39].
Meanwhile, autogenous bone grafting is considered the gold standard to repair bone defects, but some serious limitations such as donor site morbidity and limited bone supplies cannot be overcome [40]. Thus, it is necessary to develop new approaches to stimulate bone regeneration and reverse bone loss and thus treat osteoporotic defects without side effects. In our present study, we demonstrated that hiPSC-MSC-Exos effectively stimulated the proliferation and osteogenic differentiation of rBMSCs-OVX with the effect increasing with increasing concentrations of exosomes and increasing time. We also showed that the application of hiPSC-MSC-Exos promotes bone regeneration in critical-sized calvarial defects through enhanced angiogenesis and osteogenesis in an ovariectomized rat model.

In recent years, the implantation of stem cells has been an alternative option for the treatment of bone defects. MSCs are considered the "gold standard" stem cells for autologous transplantation therapy, and consequently they possess an appealing ability for bone regenerative treatments such as osteogenic differentiation [41-43]. However, harvesting adult MSCs requires suitable donors and invasive procedures [44]. In addition, the extraction and the limited cell quantity of MSCs as well as the decrease in proliferation and differentiation capacity of these cells with aging limits their therapeutic efficacy [42]. 


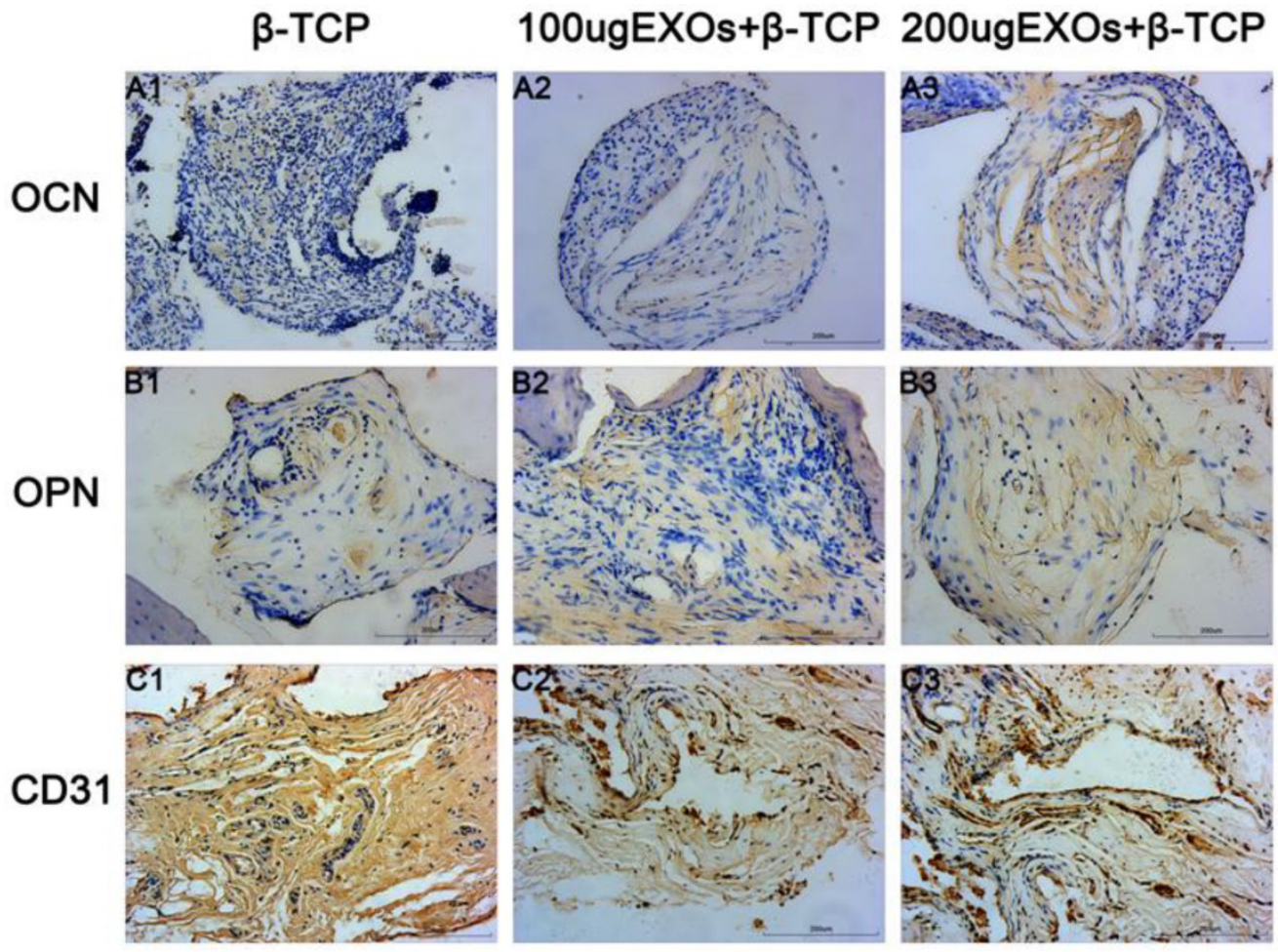

Figure 8. The immunohistochemical analysis of hiPSC-MSC-Exos $+\beta-T C P$ scaffolds promoted bone regeneration and angiogenesis. Immunohistochemical analysis of OCN $(\mathrm{A} 1-\mathrm{A} 3), \mathrm{OPN}(\mathrm{B} 1-\mathrm{B} 3)$ and $\mathrm{CD} 31(\mathrm{Cl}-\mathrm{C} 3)$ were used to detect osteogenesis and angiogenesis in specimens. The brown color represents positive staining of OCN, OPN and CD31. (Scale bar: $200 \mu \mathrm{m})$.

A recent study revealed that iPS-MSCs could be used as a cell source of adult MSCs for bone regenerative therapies [45]. Hong and colleagues [46] reported that when iPS-MSCs mixed with TCP ceramic particles were implanted into autologous macaques, new bone formation was observed. In another study, Tang [47] indicated that iPS-MSCs cultured on calcium phosphate scaffolds showed successful osteogenic differentiation in osteogenic medium. In another study [19], iPS-MSCs were implanted into immunocompromised mice with calvarial defects, and 8 weeks later, iPS-MSCs were confirmed to have participated in bone regeneration. However, the direct use of stem cells remains limited by issues of potential immunological rejection, chromosomal variation, and other problems [48]. Exosomes are considered to be an important mode of intercellular communication. The therapeutic effects of exosomes have been investigated intensively in various disease models, and results revealed that they can modulate immune responses [49], reduce the size of myocardial infarctions [50], facilitate the repair of traumatic brain injury [51] and stimulate skeletal muscle regeneration [52]. Recently, our teams have found that hiPS-MSC-Exos can effectively promote cutaneous wound healing [32]. The potential mechanism of these effects could be caused by the roles of the growth factors, cytokines, chemokines, proteins, mRNAs, and miRNAs released from exosomes [33,53]. Thus, our research aimed to investigate the influence of hiPSC-MSC-Exos on the proliferation and osteogenic differentiation of rBMSCs-OVX, and to study the effect of hiPSC-MSC-Exos on bone regeneration in critical size bone defects in an ovariectomized rat model.

The ability of BMSCs to undergo osteoblastic differentiation under osteoporotic conditions is significantly weakened, which ultimately leads to a delay in bone formation or nonunion [54]. It is known that it is critical to modulate the differentiation of BMSCs into osteoblasts and inhibit osteoclastogenesis to promote osteoporotic bone regeneration $[55,56]$. In the present study, we assumed that hiPSC-MSC-Exos have the ability to stimulate osteoblastic differentiation of rBMSCs-OVX in OM. The in vitro results suggested that hiPSC-MSC-Exos could effectively stimulate the proliferation of rBMSCs-OVX, with the effect increasing with increasing concentrations of exosomes and increasing time. The ALP activity and calcium nodule formation indicated by ARS staining were remarkably accelerated by hiPSC-MSC-Exos, and the results suggest that hiPSC-MSC-Exos can affect rBMSCs-OVX osteogenic differentiation over a long 
time course, and reveal that $200 \mu \mathrm{g} / \mathrm{mL}$ hiPSC-MSC-Exos had the strongest effect. OPN and COL1 are bone matrix proteins synthesized by osteoblasts, while RUNX2 is a key transcription factor required for bone formation; all act as early indicators of osteogenesis [57, 58]. Achieving increased expression of OPN, COL1 and RUNX2 as well as ALP activity and mineralization is an essential requisite for bone formation. The RT-qPCR results suggest that the expression of OPN, OCN and RUNX2 was up-regulated by hiPSC-MSC-Exos after 3 days, and was especially marked at 7 days of culture, while the expression was higher in the $200 \mu \mathrm{g} / \mathrm{mL}$ hiPSC-MSC-Exos group compared to the $100 \mu \mathrm{g} / \mathrm{mL}$ hiPSC-MSC-Exos group. The OPN, COL1 and RUNX2 protein expression levels were also analyzed by western blotting, and the results were consistent with the RT-qPCR results, showing that the expression of protein in the $200 \mu \mathrm{g} / \mathrm{mL}$ hiPSC-MSC-Exos group was the highest.

The OVX rat model is commonly applied in postmenopausal osteoporosis-related research, specifically by the use of skeletally mature OVX animals that show loss of trabecular and cortical bone density, as well as a significant decrease in biomechanical strength [59]. In this study, osteoporosis in the rat model was confirmed by $\mu-\mathrm{CT}$, and distal femora were chosen for quantitative analysis of the microstructural parameters $\mathrm{BV} / \mathrm{TV}$, Tb.N, Tb.Th and BMD, the results of quantification revealed that there was significantly difference in OVX rats compared to control rats, which confirmed the establishment of osteoporotic models in OVX rats groups. Therefore, the osteoporotic rats in OVX rats groups were used to research further for repairing critical-sized calvarial defects.

The calvarial defect is a non-load-bearing bone healing model and one of the most commonly used defect models in bone reconstruction [60]. Classical porous $\beta$-TCP scaffolds have good bone conduction performance, thus our study used this material as the carrier of hiPSC-MSC-Exos to repair bone defects.

The role of hiPSC-MSC-Exos in bone regeneration in vivo was determined by testing their ability to repair a critical-sized calvarial defect in an ovariectomized rat model. Micro-CT quantitative analysis showed that the application of hiPSC-MSC-Exos $+\beta$-TCP scaffolds could significantly improve osteogenesis in a calvarial defect model, but that $200 \mu \mathrm{g}$ hiPSC-MSC-Exos was more effective. HiPSC-MSC-Exos significantly enhanced new bone formation, with the efficacy increasing with increasing concentration. Histological analysis showed that there was little newly-formed bone in the defect areas in the $\beta$-TCP alone groups, whereas
hiPSC-MSC-Exos significantly promoted bone formation, and in the $200 \mu \mathrm{g}$ hiPSC-MSC-Exos $+\beta$-TCP scaffold groups bone covered most of the defect areas, and the results were consistent with the micro-CT findings. Angiogenesis is an essential step during bone healing, as restoration of blood flow provides nutrients and renewable autologous cells to heal the defect [61]. In this study, microfil perfusion was used to evaluate neovascularization at the defect site, and quantification of the percentage of new vessel area in the defects demonstrated that hiPSC-MSC-Exos treatments led to significantly more neovascularization than the $\beta$-TCP alone groups, and that the areas of newly-formed vessel increased with increasing hiPSC-MSC-Exos concentration. OCN, OPN and CD31 are common markers used in studies of osteogenic differentiation and neovascularization, respectively. Immunohistochemistry showed that there was only a small amount of positive staining for OCN, OPN and CD31 in the defect areas of the $\beta$-TCP alone group, but more positive staining for OCN, OPN and CD31 in the hiPSC-MSC-Exos group which increased with increasing exosome concentration.

In summary, all the above data demonstrated that hiPSC-MSC-Exos can improve bone regeneration of critical-sized calvarial defects in ovariectomized rats in vivo by promoting both osteogenesis and angiogenesis. Nevertheless, the detailed mechanism by which hiPS-MSC-Exos modulate rBMSCs-OVX remains incompletely understood and is being further investigated by our team, and we hope to publish the results in the near future.

\section{Conclusions}

In our present study, we have demonstrated that hiPSC-MSC-Exos effectively stimulate the proliferation and osteogenic differentiation of rBMSCs-OVX, with the effect increasing with increasing exosome concentration. Further analysis showed that the application of hiPSC-MSC-Exos $+\beta$-TCP scaffolds promoted bone regeneration in critical-sized calvarial defects through enhanced angiogenesis and osteogenesis in an ovariectomized rat model.

\section{Acknowledgements}

This study was supported by the National Nature Science Foundation of China (Grant No. 81572178).

\section{Competing Interests}

The authors have declared that no competing interest exists. 


\section{References}

1. Liu HY, Wu AT, Tsai CY, et al. The balance between adipogenesis and osteogenesis in bone regeneration by platelet-rich plasma for age-related osteoporosis. Biomaterials. 2011; 32: 6773-80.

2. Rodan GA, Martin TJ. Therapeutic approaches to bone diseases. Science. 2000; 289: 1508-14.

3. Pei L, Tontonoz P. Fat's loss is bone's gain. J Clin Invest. 2004; 113: 805-6.

4. Verron E, Gauthier O, Janvier P, et al. In vivo bone augmentation in an osteoporotic environment using bisphosphonate-loaded calcium deficient apatite. Biomaterials. 2010; 31: 7776-84.

5. Rachner TD, Khosla S, Hofbauer LC. Osteoporosis: now and the future. Lancet. 2011; 377: 1276-87.

6. Hao YJ, Zhang G, Wang YS, et al. Changes of microstructure and mineralized tissue in the middle and late phase of osteoporotic fracture healing in rats. Bone. 2007; 41: 631-8.

7. McCann RM, Colleary G, Geddis C, et al. Effect of osteoporosis on bone mineral density and fracture repair in a rat femoral fracture model. J Orthop Res. 2008; 26: 384-93.

8. Zhao S, Zhang J, Zhu $\mathrm{M}$, et al. Three-dimensional printed strontium-containing mesoporous bioactive glass scaffolds for repairing rat critical-sized calvarial defects. Acta Biomater. 2015; 12: 270-80.

9. Dimitriou R, Jones E, McGonagle D, et al. Bone regeneration: current concepts and future directions. BMC Med. 2011; 9: 66.

10. Barberi T, Willis LM, Socci ND, et al. Derivation of multipotent mesenchymal precursors from human embryonic stem cells. PLoS Med. 2005; 2: e161.

11. Chen J, Zhang ZG, Li Y, et al. Intravenous administration of human bone marrow stromal cells induces angiogenesis in the ischemic boundary zone after stroke in rats. Circ Res. 2003; 92: 692-9.

12. Pittenger MF, Mackay AM, Beck SC, et al. Multilineage potential of adult human mesenchymal stem cells. Science. 1999; 284: 143-7.

13. Katsara O, Mahaira LG, Iliopoulou EG, et al. Effects of donor age, gender, and in vitro cellular aging on the phenotypic, functional, and molecular characteristics of mouse bone marrow-derived mesenchymal stem cells. Stem Cells Dev. 2011; 20: 1549-61.

14. Barberi T, Bradbury M, Dincer Z, et al. Derivation of engraftable skeletal myoblasts from human embryonic stem cells. Nat Med. 2007; 13: 642-8.

15. Cartwright P, McLean C, Sheppard A, et al. LIF/STAT3 controls ES cell self-renewal and pluripotency by a Myc-dependent mechanism. Development. 2005; 132: 885-96.

16. Park IH, Zhao R, West JA, et al. Reprogramming of human somatic cells to pluripotency with defined factors. Nature. 2008; 451: 141-6.

17. Kang L, Wang J, Zhang Y, et al. iPS cells can support full-term development of tetraploid blastocyst-complemented embryos. Cell Stem Cell. 2009; 5: 135-8.

18. de Peppo GM, Svensson S, Lenneras M, et al. Human embryonic mesodermal progenitors highly resemble human mesenchymal stem cells and display high potential for tissue engineering applications. Tissue Eng Part A. 2010; 16: 2161-82

19. Villa-Diaz LG, Brown SE, Liu Y, et al. Derivation of mesenchymal stem cells from human induced pluripotent stem cells cultured on synthetic substrates. Stem Cells. 2012; 30: 1174-81.

20. Zou L, Luo Y, Chen M, et al. A simple method for deriving functional MSCs and applied for osteogenesis in 3D scaffolds. Sci Rep. 2013; 3: 2243.

21. Yen $\mathrm{ML}, \mathrm{Hou} \mathrm{CH}$, Peng $\mathrm{KY}$, et al. Efficient derivation and concise gene expression profiling of human embryonic stem cell-derived mesenchymal progenitors (EMPs). Cell Transplant. 2011; 20: 1529-45.

22. Zhang $\mathrm{HC}$, Liu $\mathrm{XB}$, Huang $\mathrm{S}$, et al. Microvesicles derived from human umbilical cord mesenchymal stem cells stimulated by hypoxia promote angiogenesis both in vitro and in vivo. Stem Cells Dev. 2012; 21: 3289-97.

23. Xin $\mathrm{H}$, Li Y, Buller B, et al. Exosome-mediated transfer of miR-133b from multipotent mesenchymal stromal cells to neural cells contributes to neurite outgrowth. Stem Cells. 2012; 30: 1556-64.

24. Baglio SR, Pegtel DM, Baldini N. Mesenchymal stem cell secreted vesicles provide novel opportunities in (stem) cell-free therapy. Front Physiol. 2012; 3: 359.

25. Muralidharan-Chari V, Clancy JW, Sedgwick A, et al. Microvesicles: mediators of extracellular communication during cancer progression. J Cell Sci. 2010; 123: 1603-11.

26. Pap E, Pallinger E, Pasztoi M, et al. Highlights of a new type of intercellular communication: microvesicle-based information transfer. Inflamm Res. 2009; $58: 1-8$

27. Liang X, Ding Y, Zhang Y, et al. Paracrine mechanisms of mesenchymal stem cell-based therapy: current status and perspectives. Cell Transplant. 2014; 23: 1045-59.

28. Shen $\mathrm{L}$, Zeng $\mathrm{W}, \mathrm{Wu} \mathrm{YX}$, et al. Neurotrophin-3 accelerates wound healing in diabetic mice by promoting a paracrine response in mesenchymal stem cells. Cell Transplant. 2013; 22: 1011-21.

29. Song M, Heo J, Chun JY, et al. The paracrine effects of mesenchymal stem cells stimulate the regeneration capacity of endogenous stem cells in the repair of a bladder-outlet-obstruction-induced overactive bladder. Stem Cells Dev. 2014; 23: 654-63.

30. Li T, Yan Y, Wang B, et al. Exosomes derived from human umbilical cord mesenchymal stem cells alleviate liver fibrosis. Stem Cells Dev. 2013; 22: 845-54.
31. Roccaro AM, Sacco A, Maiso P, et al. BM mesenchymal stromal cell-derived exosomes facilitate multiple myeloma progression. J Clin Invest. 2013; 123: 1542-55.

32. Zhang J, Guan J, Niu X, et al. Exosomes released from human induced pluripotent stem cells-derived MSCs facilitate cutaneous wound healing by promoting collagen synthesis and angiogenesis. J Transl Med. 2015; 13: 49.

33. Hu GW, Li O, Niu X, et al. Exosomes secreted by human-induced pluripotent stem cell-derived mesenchymal stem cells attenuate limb ischemia by promoting angiogenesis in mice. Stem Cell Res Ther. 2015; 6: 10.

34. Durao SF, Gomes PS, Colaco BJ, et al. The biomaterial-mediated healing of critical size bone defects in the ovariectomized rat. Osteoporos Int. 2014; 25: 1535-45

35. Sayed M, Drummond CA, Evans KL, et al. Effects of Na/K-ATPase and its ligands on bone marrow stromal cell differentiation. Stem Cell Res. 2014; 13: $12-23$

36. Ding H, Gao YS, Wang Y, et al. Dimethyloxaloylglycine increases the bone healing capacity of adipose-derived stem cells by promoting osteogenic differentiation and angiogenic potential. Stem Cells Dev. 2014; 23: 990-1000.

37. Lin K, Xia L, Li H, et al. Enhanced osteoporotic bone regeneration by strontium-substituted calcium silicate bioactive ceramics. Biomaterials. 2013; 34: 10028-42.

38. Zou D, Zhang Z, He J, et al. Blood vessel formation in the tissue-engineered bone with the constitutively active form of HIF-1alpha mediated BMSCs. Biomaterials. 2012; 33: 2097-108.

39. Cheng N, Dai J, Cheng X, et al. Porous CaP/silk composite scaffolds to repair femur defects in an osteoporotic model. J Mater Sci Mater Med. 2013; 24: 1963-75.

40. Monaco E, Bionaz M, Hollister SJ, et al. Strategies for regeneration of the bone using porcine adult adipose-derived mesenchymal stem cells. Theriogenology. 2011; 75: 1381-99.

41. Pei M, Li J, McConda DB, et al. A comparison of tissue engineering based repair of calvarial defects using adipose stem cells from normal and osteoporotic rats. Bone. 2015; 78: 1-10.

42. Izadpanah R, Kaushal D, Kriedt C, et al. Long-term in vitro expansion alters the biology of adult mesenchymal stem cells. Cancer Res. 2008; 68: 4229-38.

43. Blery P, Corre P, Malard $\mathrm{O}$, et al. Evaluation of new bone formation in irradiated areas using association of mesenchymal stem cells and total fresh bone marrow mixed with calcium phosphate scaffold. J Mater Sci Mater Med. 2014; 25: 2711-20.

44. Robey PG. Cell sources for bone regeneration: the good, the bad, and the ugly (but promising). Tissue Eng Part B Rev. 2011; 17: 423-30.

45. Sun $Y Q$, Deng $M X, H e ~ J$, et al. Human pluripotent stem cell-derived mesenchymal stem cells prevent allergic airway inflammation in mice. Stem Cells. 2012; 30: 2692-9.

46. Hong SG, Winkler T, Wu C, et al. Path to the clinic: assessment of iPSC-based cell therapies in vivo in a nonhuman primate model. Cell Rep. 2014; 7: 1298-309.

47. Tang M, Chen W, Liu J, et al. Human induced pluripotent stem cell-derived mesenchymal stem cell seeding on calcium phosphate scaffold for bone regeneration. Tissue Eng Part A. 2014; 20: 1295-305.

48. Collino F, Bruno S, Incarnato D, et al. AKI Recovery Induced by Mesenchymal Stromal Cell-Derived Extracellular Vesicles Carrying MicroRNAs. J Am Soc Nephrol. 2015; 26: 2349-60

49. Rahman MJ, Regn D, Bashratyan R, et al. Exosomes released by islet-derived mesenchymal stem cells trigger autoimmune responses in NOD mice. Diabetes. 2014; 63: 1008-20.

50. Lai RC, Arslan F, Lee MM, et al. Exosome secreted by MSC reduces myocardial ischemia/reperfusion injury. Stem Cell Res. 2010; 4: 214-22.

51. Zhang $\mathrm{Y}$, Chopp M, Meng $\mathrm{Y}$, et al. Effect of exosomes derived from multipluripotent mesenchymal stromal cells on functional recovery and neurovascular plasticity in rats after traumatic brain injury. J Neurosurg. 2015; 122: 856-67.

52. Nakamura $\mathrm{Y}$, Miyaki S, Ishitobi $\mathrm{H}$, et al. Mesenchymal-stem-cell-derived exosomes accelerate skeletal muscle regeneration. FEBS Lett. 2015; 589: $1257-65$

53. Kwon HM, Hur SM, Park KY, et al. Multiple paracrine factors secreted by mesenchymal stem cells contribute to angiogenesis. Vasc Pharmacol. 2014;63:19-28

54. Saidak Z, Hay E, Marty C et al Strontium ranelate rebalances bone marrow adipogenesis and osteoblastogenesis in senescent osteopenic mice through NFATc/Maf and Wnt signaling. Aging Cell. 2012; 11: 467-74.

55. Peng S, Liu XS, Wang T, et al. In vivo anabolic effect of strontium on trabecular bone was associated with increased osteoblastogenesis of bone marrow stromal cells. J Orthop Res. 2010; 28: 1208-14

56. Neve A, Cantatore FP, Corrado A, et al. In vitro and in vivo angiogenic activity of osteoarthritic and osteoporotic osteoblasts is modulated by VEGF and vitamin D3 treatment. Regul Pept. 2013; 184: 81-4

57. Wang T, Yang X, Qi X, et al. Osteoinduction and proliferation of bone-marrow stromal cells in three-dimensional poly (epsilon-caprolactone)/ hydroxyapatite/collagen scaffolds. J Transl Med. 2015; 13: 152.

58. Komori T. Requisite roles of Runx 2 and $\mathrm{Cbfb}$ in skeletal development. J Bone Miner Metab. 2003: 21: 193-7.

59. Gomes PS, Fernandes MH. Rodent models in bone-related research: the relevance of calvarial defects in the assessment of bone regeneration strategies. Lab Anim. 2011; 45: 14-24. 
60. Spicer PP, Kretlow JD, Young S, et al. Evaluation of bone regeneration using the rat critical size calvarial defect. Nat Protoc. 2012; 7: 1918-29.

61. He YX, Zhang G, Pan XH, et al. Impaired bone healing pattern in mice with ovariectomy-induced osteoporosis: A drill-hole defect model. Bone. 2011; 48: 1388-400. 